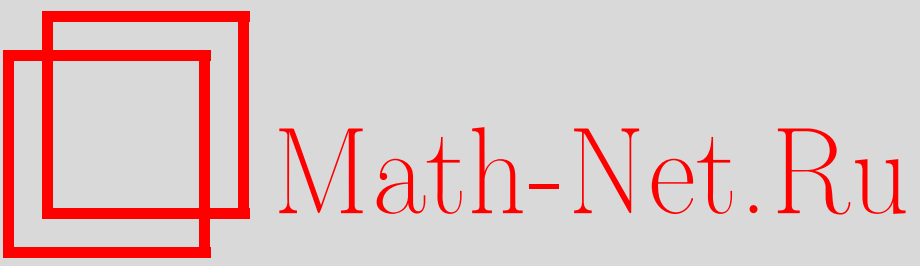

М. Л. Клепцына, А. Л. Пятницкий, Усреднение случайной нестационарной задачи конвекции-диффузии, УМH, 2002, том 57, выпуск 4, 95-118

DOI: https://doi.org/10.4213/rm535

Использование Общероссийского математического портала Math-Net.Ru подразумевает, что вы прочитали и согласны с пользовательским соглашением

http://www.mathnet.ru/rus/agreement

Параметры загрузки:

IP : 54.166 .219 .16

26 апреля 2023 г., 14:30:54 


\title{
УСРЕДНЕНИЕ СЛУЧАЙНОЙ НЕСТАЦИОНАРНОЙ ЗАДАЧИ КОНВЕКЦИИ-ДИФФУЗИИ
}

\author{
М. Л. КЛЕПЦЫнА, А. Л. ПяТнИцКИй
}

Рассматривается задача усреднения для нестационарного уравнения типа конвекции-диффузии с быстроосциллирующими коэффициентами, периодическими по пространственным переменным и случайными стационарньми по времени. В предположении, что коэффициенты уравнения обладают достаточно хорошими свойствами перемешивания, показано, что в правильно выбранных движущихся координатах распределение решения исходного уравнения сходится к решению предельного стохастического уравнения в частных производных. При этом усредненная задача корректна и определяет предельную меру однозначно.

Библиография: 25 названий.

\section{СОДЕРЖАНИЕ}

$\S 1$. Введение

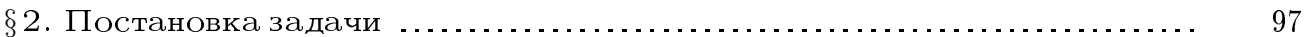

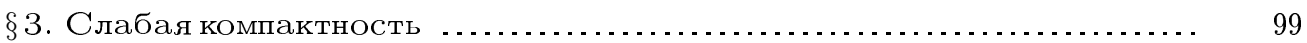

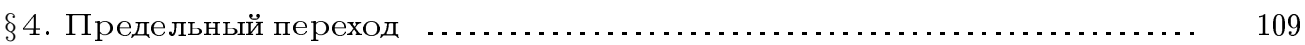

$\S 5$. Операторы с дифффузионными коэфффициентами ................... 115

Список литературы ............................................... 117

\section{$\S$ 1. Введение}

В работе изучается задача усреднения нестационарного уравнения типа конвекции-диффузии с быстроосциллируюшими коэффищиентами, которые периодичны по пространственньм переменньм и случайны по времени. Будем предполагать, что зависимость коэффициентов от времени сводится к зависимости от стационарного случайного процесса $\xi$. со значениями в $\mathbb{R}^{d}, d \geqslant 1$. Соответствующая задача Коши принимает вид

$$
\begin{gathered}
\frac{\partial}{\partial t} u^{\varepsilon}-\frac{\partial}{\partial x_{i}} a_{i j}\left(\frac{x}{\varepsilon}, \xi_{\frac{t}{\varepsilon^{2}}}\right) \frac{\partial}{\partial x_{j}} u^{\varepsilon}-\frac{1}{\varepsilon} b_{i}\left(\frac{x}{\varepsilon}, \xi_{\frac{t}{\varepsilon^{2}}}\right) \frac{\partial}{\partial x_{i}} u^{\varepsilon}=0 \\
u^{\varepsilon}(x, 0)=u_{0}(x)
\end{gathered}
$$

и мы будем исследовать предельное поведение ее решений $u^{\varepsilon}$, когда $\varepsilon \downarrow 0$.

Работа выполнена при поддержке INTAS (грант № 99-559) и РФФИ (грант № 00-01-22000). 
Ранее близкие задачи для параболических уравнений с симметрической эллиптической частью и с диффузионным процессом $\xi$. были рассмотрены в работах [7] и [13]. В [13] было показано, что для операторов в дивергентной форме справедлив классический результат теории усреднения, т.е. решения задачи Коши или начально-краевой задачи для исходного уравнения сходятся почти наверное (п.н.) при $\varepsilon \rightarrow 0$ к решению соответствуюшей задачи для усредненного параболического уравнения с постоянными неслучайными коэффициентами.

Картина предельного поведения решений сушественно меняется в присутствии растушего члена нулевого порядка, см. [7]. В этом случае предельная динамика остается, вообще говоря, случайной и результат об усреднении справедлив в ослабленной форме. А именно, семейство вероятностных мер, порожденных распределением решений исходной задачи в подходяшем функциональном пространстве, сходится слабо при $\varepsilon \rightarrow 0$ к мере, являющейся решением предельной мартингальной проблемы.

Задачи усреднения для различных эллиптических и стационарных параболических операторов с младшими членами рассматривались в работах [14], [15], [24], [19], [21]. Особое внимание в литературе было уделено операторам с несжимаемым векторным полем, здесь мы укажем работы [2], [3], [9], [10], [25].

В работе [11] изучались нестационарные параболические уравнения типа конвекции-диффузии с коэф фициентами, периодическими как по пространственным переменным, так и по времени, причем результат об усреднении был получен в предположении, что осцилляция по времени “медленнее”, чем по пространству.

Основные концепции и методы теории усреднения изложены, например, в книгах [4] и $[12]$.

Как и в неслучайной модели, при изучении задачи (1) необходимо принимать во внимание растушую как $1 / \varepsilon$ конвекцию, которая присутствует в асимптотике решения $u^{\varepsilon}$. В связи с этим результат об усреднении задачи (1) будет получен в движушихся координатах $x^{\prime}=x-\bar{b} t / \varepsilon$ с некоторым постоянным вектором $\bar{b}$. Такая замена переменных позволяет избавиться от растушего поля скоростей (порядка $1 / \varepsilon$ ) в эфифективной динамике.

В данной работе мы покажем, что семейство мер, определяемых законом распределения решения задачи (1), слабо сходится при $\varepsilon \rightarrow 0$ к решению предельной мартингальной проблемы в энергетическом функциональном пространстве. При этом диффузия, появляющаяся в предельном уравнении, конечномерна и имеет коэффициент вида $\Lambda \nabla u$, где $\Lambda=\left\{\Lambda^{i j}\right\}$ - постоянная матрица, а оператор переноса представляет собой эллиптический оператор второго порядка с постоянными коэффициентами. Матрица $\Lambda$ заслуживает особого внимания, поскольку в случае диффузионного процесса $\xi_{s}$ ее не удается построить с помошью решения обычной “локальной” задачи на ячейке с использованием генератора процесса $\xi_{s}$ и для ее построения требуется более сложнњй анализ.

В статье мы построим несколько корректоров, являюшихся решениями вспомогательных уравнений в частных производных, докажем априорные оценки и будем затем комбинировать это с идеями, развитыми в [23], [6]. Следует отметить, что некоторые из корректоров, построенных в работе, зависят не только от значения процесса $\xi_{s}$ в текущий момент времени, но и от всего “будущего" этого процесса, что составляет сушественную разницу с методами, применяемыми в [7], [13], [6], где была использована диффузионная природа корректоров. 
В $\S 2$ мы даем полную постановку задачи и формулируем все условия на коэффициенты и процесс $\xi_{s}$.

Цель $\S 3$ - доказательство слабой компактности семейства распределений решений исходной задачи.

В $\S 4$ мы вьполняем предельньй переход, строим коэффициенты предельной мартингальной проблемы и далее, благодаря единственности решения предельной задачи, обосновываем сходимость распределений $u^{\varepsilon}$.

Последний параграф̆ посвящен частному случаю, когда процесс $\xi_{s}$ является дифифузионным.

\section{§. Постановка задачи}

Мы изучаем асимптотическое поведение при малых $\varepsilon>0$ решений следуюшей задачи Коши:

$$
\begin{gathered}
\frac{\partial}{\partial t} u^{\varepsilon}(x, t)-\frac{\partial}{\partial x_{i}} a_{i j}\left(\frac{x}{\varepsilon}, \xi_{\frac{t}{\varepsilon^{2}}}\right) \frac{\partial}{\partial x_{j}} u^{\varepsilon}(x, t)-\frac{1}{\varepsilon} b_{i}\left(\frac{x}{\varepsilon}, \xi_{\frac{t}{\varepsilon^{2}}}\right) \frac{\partial}{\partial x_{i}} u^{\varepsilon}(x, t)=0, \\
u^{\varepsilon}(x, 0)=u_{0}(x)
\end{gathered}
$$

здесь $\xi_{s}$ - это эргодический стационарньй случайный процесс, заданньй на вероятностном пространстве $(\Omega, \mathscr{F}, \mathrm{P})$. Символом $\mathrm{E}$ будет обозначаться математическое ожидание. В случае, когда $\xi_{s}$ диффузионньй процесс, будем обозначать через $L$ его инфинитезимальньй генератор:

$$
L=q_{i j}(y) \frac{\partial}{\partial y_{i}} \frac{\partial}{\partial y_{j}}+B_{i}(y) \frac{\partial}{\partial y_{i}} .
$$

Другие условия на процесс $\xi_{s}$ будут заданы в терминах коэффициентов равномерного или сильного перемешивания либо коэффициента максимальной коррелящии. Для удобства читателя мы здесь даем соответствуюшие определения.

Пусть $\mathscr{F} \leqslant t$ и $\mathscr{F} \geqslant t$ - это $\sigma$-алгебры $\sigma\left(\xi_{s}, s \leqslant t\right)$ и $\sigma\left(\xi_{s}, s \geqslant t\right)$ соответственно. Функция $\alpha(s), s \geqslant 0$, задаваемая соотношением

$$
\alpha(s)=\sup _{\mathscr{B}_{1} \in \mathscr{F}_{F} \leqslant 0, \mathscr{B}_{2} \in \mathscr{F}_{\geqslant s}}\left|\mathrm{P}\left(\mathscr{B}_{1} \cup \mathscr{B}_{2}\right)-\mathrm{P}\left(\mathscr{B}_{1}\right) \mathrm{P}\left(\mathscr{B}_{2}\right)\right|,
$$

называется коэффициентом сильного перемешивания процесса $\{\xi$.$\} . Функция \phi(s)$, $s \geqslant 0$,

$$
\phi(s)=\sup _{\mathscr{B}_{1} \in \mathscr{F}_{\leqslant 0}, \mathscr{B}_{2} \in \mathscr{F}_{\geqslant} \geqslant s}\left|\mathrm{P}\left(\mathscr{B}_{1} \mid \mathscr{B}_{2}\right)-\mathrm{P}\left(\mathscr{B}_{1}\right)\right|
$$

называется коэффициентом равномерного перемешивания $\{\xi$. $\}$. Наконец, коэфффициент максимальной корреляции $\rho(s), s \geqslant 0$, определяется равенством

$$
\rho(s)=\sup \frac{\left|\operatorname{cov}\left(\eta_{1}, \eta_{2}\right)\right|}{\sqrt{\mathrm{E}\left(\eta_{1}-\mathrm{E}\left(\eta_{1}\right)\right)^{2} \mathrm{E}\left(\eta_{2}-\mathrm{E}\left(\eta_{2}\right)\right)^{2}}},
$$


в котором sup берется по всем $\mathscr{F} \leqslant 0$-измеримым $\eta_{1}$ и $\mathscr{F} \geqslant s$-измеримым $\eta_{2}$ таким, что $\mathrm{E}\left(\eta_{1}\right)^{2}<\infty$ и $\mathrm{E}\left(\eta_{2}\right)^{2}<\infty$; обозначение cov используется для ковариации.

Мы всегда предполагаем, что коэффициенты $a_{i j}(z, y)$ и $b_{i}(z, y)$ периодичны по переменной $z$. Остальные предположения сформулированы ниже.

A1. Функции $a_{i j}(z, y)$ и $b_{i}(z, y)$ и их первые производные по $z$ и $y$ равномерно ограничены:

$$
\begin{gathered}
\left|a_{i j}(z, y)\right|+\left|\nabla_{z} a_{i j}(z, y)\right|+\left|\nabla_{y} a_{i j}(z, y)\right| \leqslant c \\
\left|b_{i}(z, y)\right|+\left|\nabla_{z} b_{i}(z, y)\right|+\left|\nabla_{y} b_{i}(z, y)\right| \leqslant c
\end{gathered}
$$

здесь и далее символом с обозначаются положительные константы.

A2. Матрица $a_{i j}(z, y)$ положительно определена:

$$
a_{i j} \eta_{i} \eta_{j} \geqslant c|\eta|^{2}, \quad \eta \in \mathbb{R}^{n}
$$

для некоторого $c>0$ при всех $(z, y) \in \mathbb{T}^{n} \times \mathbb{R}^{d}$.

A3. Выполнено хотя бы одно из следуюших условий:

$$
\int_{0}^{\infty}(\alpha(s))^{1 / 2} d s<\infty ; \quad \int_{0}^{\infty}(\phi(s))^{1 / 2} d s<\infty ; \quad \int_{0}^{\infty} \rho(s) d s<\infty .
$$

В частном случае, когда процесс $\xi_{s}$ диффузионный, возникает естественное желание заменить предположение $\mathbf{A 3}$ некоторым его эффективно проверяемым достаточным условием, формулируемым в терминах коэффициентов генератора процесса. Однако, как оказалось, существенно удобнее использовать для этой цели не генератор исходного процесса $\xi_{s}$, а генератор процесса с обрашенным временем $\zeta_{s}=\xi_{-s}$. В связи с этим мы вводим обозначение $\widetilde{L}$ для генератора $\zeta_{s}$,

$$
\widetilde{L}=\widetilde{q}_{k l}(y) \frac{\partial}{\partial y_{k}} \frac{\partial}{\partial y_{l}}+\widetilde{B}_{k}(y) \frac{\partial}{\partial y_{k}},
$$

и предполагаем, что вместо $\mathbf{A} \mathbf{3}$ выполнено следующее условие.

$\mathbf{A 3}^{\prime}$. Коэффициенты диффузии $\widetilde{q}_{k l}(y)$ и их производные первого порядка равномерно ограничены:

$$
\left|\widetilde{q}_{k l}(y)\right|+\left|\nabla_{y} \widetilde{q}_{k l}(y)\right| \leqslant c
$$

оператор $\widetilde{L}$ равномерно эллиптичен:

$$
\widetilde{q}_{k l} \eta_{k} \eta_{l} \geqslant c|\eta|^{2}, \quad \eta \in \mathbb{R}^{d},
$$

при всех $y \in \mathbb{R}^{d}$. Вектор-функция $\widetilde{B}(y)$ допускает полиномиальную оценку

$$
|\widetilde{B}(y)|+|\nabla \widetilde{B}(y)| \leqslant c\left(1+|y|^{\kappa}\right)
$$

с некоторьм $\kappa>0$ и, кроме того, сушествуют такие $\mu>-1, R>0$ и $c>0$, что при всех $y$ из $\{y:|y| \geqslant R\}$ выполнено неравенство

$$
\frac{\widetilde{B}(y) \cdot y}{|y|} \leqslant-c|y|^{\mu}
$$


Согласно [20] (см. также [7]) при выполнении условия $\mathbf{A} \mathbf{3}^{\prime}$ процесс $\zeta_{s}$ (а следовательно, и $\xi_{s}$ ) обладает единственной инвариантной мерой, причем эта мера имеет непрерьвную плотность $\rho(y)$, удовлетворяющую уравнению

$$
\widetilde{L}^{*} \rho=0, \quad \int_{\mathbb{R}^{d}} \rho(y) d y=1,
$$

и убьвающую на бесконечности быстрее любой отрицательной степени $|y|$; обозначение $\widetilde{L}^{*}$ здесь использовано для сопряженного с $\widetilde{L}$ оператора. $\mathrm{K}$ тому же для стационарной версии процесса $\zeta_{s}$ с генератором $\widetilde{L}$ коэффициент сильного перемешивания $\alpha(s)$ убывает на бесконечности быстрее любой отрицательной степени $s$, что влечет условие $\mathbf{A 3 .}$

Далее, распределение стационарного процесса $\xi_{s}=\zeta_{-s}$ в пространстве траекторий совпадает с распределением стационарного диффузионного процесса с генератором

$$
L=(\rho(y))^{-1} \widetilde{L}^{*}(\rho(y) \cdot)=\widetilde{q}_{k l}(y) \frac{\partial}{\partial y_{k}} \frac{\partial}{\partial y_{l}}+\left((\rho(y))^{-1} \frac{\partial}{\partial y_{i}}\left[\rho(y) \widetilde{q}_{k i}(y)\right]-\widetilde{B}_{k}(y)\right) \frac{\partial}{\partial y_{k}} ;
$$

в дальнейшем мы отождествляем эти процессы.

В предположениях $\mathbf{A} \mathbf{1}-\mathbf{A} \mathbf{3}$ при каждом начальном условии $u_{0} \in L^{2}\left(\mathbb{R}^{n}\right)$ и каждом $\varepsilon>0$ задача (2) имеет почти неверное (п.н.) единственное решение

$$
u^{\varepsilon} \in L^{2}\left(0, T ; H^{1}\left(\mathbb{R}^{n}\right)\right) \cup C\left(0, T ; L^{2}\left(\mathbb{R}^{n}\right)\right) .
$$

Распределение этого решения порождает вероятностную меру Радона в пространстве

$$
V=L_{w}^{2}\left(0, T ; H^{1}\left(\mathbb{R}^{n}\right)\right) \cup C\left(0, T ; L_{w}^{2}\left(\mathbb{R}^{n}\right)\right)
$$

с борелевской $\sigma$-алгеброй, символ $w$ здесь указывает на слабую топологию. Эта мера, определяемая как распределение $u^{\varepsilon}$ в $V$, будет обозначаться $Q^{\varepsilon}$.

\section{§3. Слабая компактность}

В этом параграфе мы устанавливаем результаты о компактности для семейства мер $Q^{\varepsilon}$. В общем случае само это семейство мер не является слабо компактным в $V$. Для того чтобы получить компактное семейство, мы вводим движущуюся систему координат $\left(x^{\prime}, t\right)=(x-\bar{b} / \varepsilon, t)$ с постоянньм вектором $\bar{b}$ и показьваем, что при правильном выборе $\bar{b}$ семейство распределений функций $u^{\varepsilon}\left(x^{\prime}, t\right)$ будет слабо компактным в $V$. Этот результат опирается на априорные оценки для решения задачи (2) и теорему Прохорова (см. [23], [6]).

ПРеДЛОЖениЕ 1. Равномерно по $\varepsilon>0$ выполнена оценка

$$
\sup _{t \leqslant T}\left(\left\|u^{\varepsilon}(t)\right\|_{L^{2}\left(\mathbb{R}^{n}\right)}^{2}+\int_{0}^{t}\left\|\nabla u^{\varepsilon}(s)\right\|_{L^{2}\left(\mathbb{R}^{n}\right)}^{2} d s\right) \leqslant c\left\|u_{0}\right\|_{L^{2}\left(\mathbb{R}^{n}\right)}^{2} .
$$

Существует постоянныц вектор $\bar{b}$ такой, что для любых $\varphi(x) \in C_{0}^{\infty}\left(\mathbb{R}^{n}\right)$ u $\gamma>0$ имеем

$$
\lim _{\nu \rightarrow 0} \sup _{\varepsilon>0} \mathrm{P}\left\{\sup _{|t-s|<\nu}\left|\left(u^{\varepsilon}(t), \varphi\left(\cdot+\frac{\bar{b}}{\varepsilon} t\right)\right)-\left(u^{\varepsilon}(s), \varphi\left(\cdot+\frac{\bar{b}}{\varepsilon} s\right)\right)\right| \geqslant \gamma\right\}=0 .
$$


ДокаЗАТЕЛЬСтво. Рассмотрим вспомогательную задачу

$$
\begin{gathered}
\frac{\partial}{\partial s} p_{\varepsilon}(z, s)+A^{*} p_{\varepsilon}(z, s)=0, \quad(z, t) \in \mathbb{T}^{n} \times\left(-\infty,(T+1) / \varepsilon^{2}\right), \\
\left.p_{\varepsilon}\right|_{s=(T+1) / \varepsilon^{2}}=1,
\end{gathered}
$$

где

$$
A^{*}=\frac{\partial}{\partial z_{i}} a^{i j}\left(z, \xi_{s}\right) \frac{\partial}{\partial z_{j}}-\frac{\partial}{\partial z_{i}}\left(b^{i}\left(z, \xi_{s}\right) \cdot\right) .
$$

По принципу максимума (см. [16]) решение $p_{\varepsilon}(z, s)$ строго положительно. Более того, учитьвая структуру уравнения и интегрируя по частям на множестве $\mathbb{T}^{n} \times$ $\left(s,(T+1) / \varepsilon^{2}\right)$, получим

$$
\int_{\mathbb{T}^{n}} p_{\varepsilon}(z, s) d z=1
$$

при всех $s \leqslant(T+1) / \varepsilon^{2}$. С помошью неравенства Гарнака (см. [22]) имеем

$$
0<c_{1} \leqslant p_{\varepsilon}(z, s) \leqslant c_{2}<\infty,
$$

причем константы $c_{1}$ и $c_{2}$ не зависят от $\varepsilon$ и от траектории $\xi_{s}$.

Если теперь умножить уравнение (2) на $p_{\varepsilon}\left(x / \varepsilon, s / \varepsilon^{2}\right) u^{\varepsilon}(x, s)$ и проинтегрировать результат по множеству $\mathbb{R}^{n} \times(0, t)$, то после простых преобразований получаем

$$
\begin{aligned}
& \frac{1}{2} \int_{\mathbb{R}^{n}}\left(u^{\varepsilon}(x, t)\right)^{2} p_{\varepsilon}\left(\frac{x}{\varepsilon}, \frac{t}{\varepsilon^{2}}\right) d x-\frac{1}{2} \int_{\mathbb{R}^{n}}\left(u_{0}(x, t)\right)^{2} p_{\varepsilon}\left(\frac{x}{\varepsilon}, 0\right) d x \\
& \quad+\int_{0}^{t} \int_{\mathbb{R}^{n}} a_{i j}\left(\frac{x}{\varepsilon}, \xi_{s / \varepsilon^{2}}\right) p_{\varepsilon}\left(\frac{x}{\varepsilon}, \frac{s}{\varepsilon^{2}}\right) \frac{\partial}{\partial x_{i}} u^{\varepsilon}(x, s) \frac{\partial}{\partial x_{j}} u^{\varepsilon}(x, s) d x d s \\
& \quad-\frac{1}{2} \int_{0}^{t} \int_{\mathbb{R}^{n}}\left(u^{\varepsilon}\right)^{2}\left[\frac{\partial}{\partial t} p_{\varepsilon}\left(\frac{x}{\varepsilon}, \frac{s}{\varepsilon^{2}}\right)+\frac{\partial}{\partial x_{i}} \frac{\partial}{\partial x_{j}}\left(a_{i j}\left(\frac{x}{\varepsilon}, \xi_{s / \varepsilon^{2}}\right) p_{\varepsilon}\left(\frac{x}{\varepsilon}, \frac{s}{\varepsilon^{2}}\right)\right)\right. \\
& \left.\quad-\frac{\partial}{\partial x_{i}}\left(b_{i}\left(\frac{x}{\varepsilon}, \xi_{s / \varepsilon^{2}}\right) p_{\varepsilon}\left(\frac{x}{\varepsilon}, \frac{s}{\varepsilon^{2}}\right)\right)\right] d x d s=0 .
\end{aligned}
$$

Согласно (5) последний интеграл здесь равен 0, поэтому с учетом (7) легко вьводим (3).

Переходим теперь к (4). Ключевым здесь является следуюшее утверждение.

Лемма 1. Пусть начальное условие $u_{0}(x)$ в (2) есть функиия класса $C_{0}^{\infty}$. Тогда существует неслучайная функщия $\kappa_{1}(\varepsilon)$, стремящаяся $к$ нулю при $\varepsilon \downarrow 0$, такая, что

$$
\sup _{(x, t) \in \mathbb{R}^{n} \times(0, T)} \underset{G_{x}^{\varepsilon}}{\operatorname{osc}} u^{\varepsilon} \leqslant \kappa_{1}(\varepsilon) ;
$$

здесь $G_{x}^{\varepsilon}=x+(-\varepsilon / 2, \varepsilon / 2)^{n}$. 
ДокАЗАТЕЛЬСтво. Рассмотрим вспомогательную периодическую задачу Коши

$$
\begin{gathered}
\frac{\partial}{\partial s} v(z, s)-A v(z, s)=0, \quad z \in \mathbb{T}^{n}, \quad s>s_{0}, \\
\left.v\right|_{s=s_{0}}=v_{0}
\end{gathered}
$$

с оператором

$$
A=\frac{\partial}{\partial z_{i}} a^{i j}\left(z, \xi_{s}\right) \frac{\partial}{\partial z_{j}}+b^{i}\left(z, \xi_{s}\right) \frac{\partial}{\partial z_{i}} .
$$

Ее решение $v(z, s)$ сходится с экспоненциальной скоростью к константе при $s-s_{0} \rightarrow \infty$. В частности,

$$
\underset{\mathbb{T}^{n}}{\operatorname{osc}} v\left(\cdot, s_{0}+\varepsilon^{-1 / 2}\right) \leqslant c^{\prime} \exp \left(-c \varepsilon^{-1 / 2}\right)\left|v_{0}\right|_{L^{\infty}}
$$

причем $c^{\prime}$ и $c$ не зависят от $s_{0}$ и от реализации $\xi$. . Действительно, в предположениях $\mathbf{A 1}$ и $\mathbf{A 2}$ решение $v(z, s)$ удовлетворяет равномерному неравенству Гарнака, которое, в свою очередь, обеспечивает требуемую оценку (см., например, доказательство леммы 2 ниже).

Оператор

$$
A^{\varepsilon}=\frac{\partial}{\partial x_{i}} a_{i j}\left(\frac{x}{\varepsilon}, \xi_{\frac{t}{\varepsilon^{2}}}\right) \frac{\partial}{\partial x_{j}}+\frac{1}{\varepsilon} b_{i}\left(\frac{x}{\varepsilon}, \xi_{\frac{t}{\varepsilon^{2}}}\right) \frac{\partial}{\partial x_{i}}
$$

коммутирует с любым сдвигом вида $S_{k} u(x)=u(x+\varepsilon k), k \in \mathbb{Z}^{n}$, поэтому с помошюю принципа максимума имеем

$$
\left|u^{\varepsilon}(x+\varepsilon k, t)-u^{\varepsilon}(x, t)\right| \leqslant M \varepsilon|k|, \quad k \in \mathbb{Z}^{n},
$$

где $M=\max _{x}\left|\nabla u_{0}(x)\right|$.

Далее, мы выбираем произвольные $t_{0} \geqslant 0$ и $x_{0} \in \mathbb{R}^{n}$, сужаем функцию $u^{\varepsilon}\left(x, t_{0}\right)$ на $G_{x_{0}}^{\varepsilon}$ и обозначаем через $\widetilde{v}_{0}^{\varepsilon}$ периодическое продолжение этого сужения с периодом $\varepsilon$ по всем координатным направлениям. Благодаря (11) при всех $x$ таких, что $\left|x-x_{0}\right| \leqslant$ $\varepsilon^{1 / 4}$, справедлива оценка

$$
\left|\widetilde{v}_{0}^{\varepsilon}(x)-u^{\varepsilon}\left(x, t_{0}\right)\right| \leqslant M \varepsilon^{1 / 4} .
$$

Пусть $p\left(t, t^{\prime}, x, x^{\prime}\right)$ - это фундаментальное решение задачи (2). Согласно [1] в предположениях $\mathbf{A 1 - \mathbf { A 2 }}$ для всех $x, x^{\prime}$ таких, что $\left|x-x^{\prime}\right|>\varepsilon^{1 / 4}$, выполнено неравенство

$$
p\left(t_{0}, t_{0}+\varepsilon^{3 / 2}, x, x^{\prime}\right) \leqslant c^{\prime} \exp \left(-c\left|x-x^{\prime}\right|^{2} / \varepsilon^{3 / 2}\right),
$$

в котором $c$ и $c^{\prime}$ зависят только от констант из условий $\mathbf{A 1}-\mathbf{A 2}$. Интегрируя это неравенство на множестве $\left\{x:\left|x-x^{\prime}\right|>\varepsilon^{1 / 4}\right\}$, найдем

$$
\int_{\left|x-x^{\prime}\right|>\varepsilon^{1 / 4}} p\left(t_{0}, t_{0}+\varepsilon^{3 / 2}, x, x^{\prime}\right) d x \leqslant c \varepsilon .
$$


Пусть $\widetilde{v}^{\varepsilon}(x, t)$ - решение уравнения $\partial_{t} \widetilde{v}^{\varepsilon}-A^{\varepsilon} \widetilde{v}^{\varepsilon}=0$ с начальньп условием $\left.\widetilde{v}^{\varepsilon}\right|_{t=t_{0}}=$ $\widetilde{v}_{0}^{\varepsilon}(x)$. Тогда оценка (10) дает

$$
\underset{\mathbb{R}^{n}}{\operatorname{osc}} \widetilde{v}^{\varepsilon}\left(\cdot, t_{0}+\varepsilon^{3 / 2}\right) \leqslant c^{\prime} \exp \left(-c \varepsilon^{-1 / 2}\right)\left|\widetilde{v}_{0}\right|_{L^{\infty}} .
$$

С другой стороны, из (12) и (14) следует, что

$$
\left|u^{\varepsilon}\left(x, t_{0}+\varepsilon^{3 / 2}\right)-\widetilde{v}^{\varepsilon}\left(x, t_{0}+\varepsilon^{3 / 2}\right)\right| \leqslant c \varepsilon\left|u_{0}\right|_{L^{\infty}}+c M \varepsilon^{1 / 4}
$$

при всех $x \in G_{x_{0}}^{\varepsilon}$. Из последних двух неравенств вытекает требуемое утверждение при всех $t \geqslant \varepsilon^{3 / 2}$. Для малых $t$ это утверждение является элементарньм следствием оценки (14), что завершает доказательство леммы.

Далее нам понадобится последовательность задач

$$
\begin{gathered}
\frac{\partial}{\partial s} p^{N}(z, s)+A^{*} p^{N}(z, s)=0, \quad(z, s) \in \mathbb{T}^{n} \times(-\infty, N) \\
p^{N}(z, N)=1
\end{gathered}
$$

с оператором $A^{*}$, заданньм формулой (6). С помошью рассуждений, использованных вьше, легко проверить, что функции $p^{N}$ удовлетворяют равномерной по $N$ и $s$, $-\infty<s \leqslant N$, оценке $(7)$.

ЛЕмма 2. Последовательность $p^{N}$ сходится при $N \rightarrow \infty \kappa$ стационарному эргодическому прочессу со значениями в $C\left(\mathbb{T}^{n}\right)$, и реализации этого прочесса удовлетворяют уравнению

$$
\frac{\partial}{\partial s} p+A^{*} p=0, \quad \int_{\mathbb{T}^{n}} p(z, s) d z=1, \quad s \in(-\infty,+\infty) .
$$

Справедлива оченка

$$
\max _{\substack{z \in \mathbb{T}^{n} \\ k \leqslant s \leqslant k+1}}\left|p^{N}(z, s)-p(z, s)\right| \leqslant c_{1} \exp (-c(N-k))
$$

с неслучайными константами с $>0$ и $c_{1}>0$.

ДокАЗАТЕльСтво. Для обоснования сходимости и оценки (17) рассмотрим следующую задачу:

$$
\frac{\partial}{\partial s} q+A^{*} q=0,\left.\quad q\right|_{s=N}=q_{0}
$$

в которой $q_{0} \in L^{2}\left(\mathbb{T}^{n}\right)$ удовлетворяет равенству

$$
\int_{\mathbb{T}^{n}} q_{0}(z) d z=0
$$

Покажем сначала, что для нашей цели достаточно проверить экспоненциальное затухание решения задачи (18) при $N-s \rightarrow \infty$, т.е. получить оценку

$$
|q(z, s)| \leqslant c_{1}\left\|q_{0}\right\|_{L^{2}\left(\mathbb{T}^{n}\right)} \exp (-c(N-s)) .
$$


Действительно, при всех $N>0$ и $k>0$ разность $p^{N+k}-p^{N}$ есть решение уравнения

$$
\frac{\partial}{\partial s}\left(p^{N+k}-p^{N}\right)+A^{*}\left(p^{N+k}-p^{N}\right)=0, \quad s<N,
$$

и при всех $s \leqslant N$ вьполнено равенство

$$
\int_{\mathbb{T}^{n}}\left(p^{N+k}(z, s)-p^{N}(z, s)\right) d z=0, \quad\left\|p^{N+k}-p^{N}\right\|_{L^{\infty}} \leqslant c .
$$

Поэтому в предположении, что справедлива оценка (20), мы имеем

$$
\left\|p^{N+k}-p^{N}\right\|_{C\left(\mathbb{T}^{n} \times[k, k+1]\right)} \leqslant c_{1} \exp (-c(N-k)) .
$$

По критерию Коши $p^{N}$ сходится при $N \rightarrow \infty$ к предельной непрерьвной функции, обозначаемой $p(z, s)$, и для этой функции выполнено (17). Переходя к пределу в (15), приходим к (16).

Для доказательства (20) рассмотрим сопряженную задачу

$$
\begin{gathered}
\frac{\partial}{\partial s} \nu(z, s)-A \nu(z, s)=0, \quad(z, s) \in \mathbb{T}^{n} \times\left(s_{0},+\infty\right), \\
\nu\left(z, s_{0}\right)=\varphi(z),
\end{gathered}
$$

в которой $\varphi$ - произвольная $L^{2}\left(\mathbb{T}^{n}\right)$-функция. Умножив это уравнение на $q(z, s)$ и проинтегрировав его на множестве $\mathbb{T}^{n} \times\left[s_{0}, N\right]$, после нескольких интегрирований по частям, найдем

$$
\int_{\mathbb{T}^{n}} \nu(z, N) q_{0}(z) d z=\int_{\mathbb{T}^{n}} \varphi(z) q\left(z, s_{0}\right) d z .
$$

Далее, применяя оценку Нэша (см. [16]), получим

$$
\max _{z \in \mathbb{T}^{n}}\left|\nu\left(z, s_{0}+1\right)\right| \leqslant c\|\varphi\|_{L^{2}\left(\mathbb{T}^{n}\right)} .
$$

Пусть $\nu^{+}\left(z, s_{1}\right)$ и $\nu^{-}\left(z, s_{1}\right)$ - это положительная и отрицательная части $\nu\left(z, s_{1}\right)$ :

$$
\nu^{+}\left(z, s_{1}\right)=\max \left(\nu\left(z, s_{1}\right), 0\right) ; \quad \nu^{-}\left(z, s_{1}\right)=-\min \left(\nu\left(z, s_{1}\right), 0\right) .
$$

Вычитая, если необходимо, подходящую константу, мы предполагаем без ограничения обшности, что

$$
\max _{z \in \mathbb{T}^{n}} \nu\left(z, s_{1}\right)=-\min _{z \in \mathbb{T}^{n}} \nu\left(z, s_{1}\right)
$$

и, как следствие, $\left\|\nu^{+}\left(\cdot, s_{1}\right)\right\|_{L^{\infty}}=\left\|\nu^{-}\left(\cdot, s_{1}\right)\right\|_{L^{\infty}}$. Применяя неравенство Гарнака к решению задачи

$$
\begin{aligned}
\frac{\partial}{\partial s} \nu^{1}(z, s)-A \nu^{1}(z, s) & =0, \quad(z, s) \in \mathbb{T}^{n} \times\left(s_{1},+\infty\right), \\
\nu^{1}\left(z, s_{1}\right) & =\nu^{+}\left(z, s_{1}\right)
\end{aligned}
$$


получим оценку

$$
\max _{z \in \mathbb{T}^{n}} \nu^{1}\left(z, s_{1}+1\right) \leqslant c_{2} \min _{z \in \mathbb{T}^{n}} \nu^{1}\left(z, s_{1}+1\right)
$$

с константой $c_{2}$, зависящей только от постоянных из условий $\mathbf{A 1}$ и $\mathbf{A 2}$ В совокупности с тривиальной оценкой

$$
0 \leqslant \nu^{1} \leqslant\left\|\nu^{+}\left(\cdot, s_{1}\right)\right\|_{L^{\infty}}
$$

Это дает

$$
0<c_{3} \leqslant \nu^{1}\left(z, s_{1}+1\right) \leqslant\left\|\nu^{+}\left(\cdot, s_{1}\right)\right\|_{L^{\infty}} .
$$

Аналогично, решение $\nu^{2}$ задачи

$$
\frac{\partial}{\partial s} \nu^{2}(z, s)-A \nu^{2}(z, s)=0, \quad \nu^{2}\left(z, s_{1}\right)=\nu^{-}\left(z, s_{1}\right)
$$

допускает оценку

$$
0<c_{3} \leqslant \nu^{2}\left(z, s_{1}+1\right) \leqslant\left\|\nu^{-}\left(\cdot, s_{1}\right)\right\|_{L^{\infty}} .
$$

В силу последних двух оценок найдется $c_{4}>0$ такая, что при всех $s>s_{0}+1$ выполнено неравенство

$$
\underset{\mathbb{T}^{n}}{\operatorname{Osc}} \nu(\cdot, s+1) \leqslant\left(1-c_{4}\right) \underset{\mathbb{T}^{n}}{\operatorname{Osc}} \nu(\cdot, s)
$$

Отсюда с учетом (23) вьводим

$$
\underset{\mathbb{T}^{n}}{\operatorname{osc}} \nu(\cdot, s) \leqslant c_{1} \exp \left(-c\left(s-s_{0}\right)\right)\|\varphi\|_{L^{2}\left(\mathbb{T}^{n}\right)}
$$

для всех $s \geqslant s_{0}+1$. Наконец, из $(22),(19)$ и последней оценки следует, что

$\left|\int_{\mathbb{T}^{n}} \varphi(z) q\left(z, s_{0}\right) d z\right|=\left|\int_{\mathbb{T}^{n}} \nu(z, N) q_{0}(z) d z\right| \leqslant c_{1} \exp \left(-c\left(N-s_{0}\right)\right)\left\|q_{0}\right\|_{L^{2}\left(\mathbb{T}^{n}\right)}\|\varphi\|_{L^{2}\left(\mathbb{T}^{n}\right)}$

при всех $\varphi \in L^{2}\left(\mathbb{T}^{n}\right)$. Следовательно,

$$
\left\|q\left(\cdot, s_{0}\right)\right\|_{L^{2}\left(\mathbb{T}^{n}\right)} \leqslant c_{1} \exp \left(-c\left(N-s_{0}\right)\right)\left\|q_{0}\right\|_{L^{2}\left(\mathbb{T}^{n}\right)}
$$

и

$$
\left|q\left(z, s_{0}\right)\right| \leqslant c_{1} \exp \left(-c\left(N-s_{0}\right)\right)\left\|q_{0}\right\|_{L^{2}\left(\mathbb{T}^{n}\right)} ;
$$

при вьводе последнего неравенства снова была использована оценка Нэша.

Рассмотрим функции $\widetilde{p}^{N}(z, s)=p^{N+s}(z, s)$, где $p^{N}$ - это решение задачи (15). При каждом $N>0$ функция $\widetilde{p}^{N}$ определена при всех $s \in \mathbb{R}$ и представляет собой стационарный эргодический процесс со значениями в $C\left(\mathbb{T}^{n}\right)$. Как было доказано выше, при $N \rightarrow \infty$ последовательность $\widetilde{p}^{N}(z, s)$ сходится с экспоненциальной скоростью к $p(z, s)$. Следовательно, $p(\cdot, s)$ также стационарньй эргодический процесс. Лемма доказана.

Определим теперь следующий постоянньй вектор:

$$
\bar{b}^{i}=\mathrm{E} \int_{\mathbb{T}^{n}}\left(\frac{\partial}{\partial z_{j}} a^{i j}\left(z, \xi_{s}\right)+b^{i}\left(z, \xi_{s}\right)\right) p(z, s) d z
$$


и стационарный случайный процесс со значениями в $\mathbb{R}^{n}$ :

$$
\eta^{i}(s)=\int_{\mathbb{T}^{n}}\left(\frac{\partial}{\partial z_{j}} a^{i j}\left(z, \xi_{s}\right)+b^{i}\left(z, \xi_{s}\right)-\bar{b}^{i}\right) p(z, s) d z .
$$

Все процессы, участвующие в определении вектора $\bar{b}$, стационарны и эргодичны, поэтому по теореме Биркгофа имеем

$$
\bar{b}^{i}=\lim _{T \rightarrow \infty} \frac{1}{T} \int_{0}^{T} \int_{\mathbb{T}^{n}}\left(\frac{\partial}{\partial z_{j}} a^{i j}\left(z, \xi_{s}\right)+b^{i}\left(z, \xi_{s}\right)\right) p(z, s) d z d s
$$

для почти всех $\omega \in \Omega$.

ЛЕмма 3. Для процесса $\eta(s)$ выполняется функциональная иентральная предельная теорема (приниип инвариантности) с ковариачионной матрицей

$$
\left\{\sigma^{2}\right\}^{i j}=\int_{0}^{\infty} \mathrm{E}\left(\eta^{i}(s) \eta^{j}(0)+\eta^{i}(0) \eta^{j}(s)\right) d s .
$$

To ecmb

$$
\varepsilon \int_{0}^{\cdot / \varepsilon^{2}} \eta(s) d s \underset{\varepsilon \rightarrow 0}{\stackrel{\mathscr{L}}{\longrightarrow}} \sigma w .
$$

в $(C[0, T])^{n}$, әде $w$. - стандартное $n$-мерное броуновское движение.

ДокАЗАТЕльство. Прежде всего мы хотим доказать, что при всех $T>0$ вьполняется неравенство

$$
\left\|\mathrm{E}\left\{\eta_{0} \mid \mathscr{F} \geqslant T\right\}\right\|_{L^{2}(\Omega)} \leqslant c_{1}(\exp (-c T)+\rho(T / 2))
$$

с некоторыми константами $c>0$ и $c_{1}>0$. Для этого на интервале $0 \leqslant s \leqslant T / 2$ мы записьваем функцию $p(z, s)$ в виде суммы $p(z, s)=p^{1}(z, s)+p^{2}(z, s)$, в которой каждое из слагаемых $p^{1}$ и $p^{2}$ удовлетворяет уравнению

$$
\frac{\partial}{\partial s} p^{i}(z, s)+A^{*} p^{i}(z, s)=0, \quad s<T / 2
$$

с начальными условиями $\left.p^{1}\right|_{s=T / 2}=1$ и $\left.p^{2}\right|_{s=T / 2}=p(z, T / 2)-1$. Тогда $\eta(0)=$ $\eta^{1}(0)+\eta^{2}(0)$, где

$$
\eta^{m, i}(0)=\int_{\mathbb{T}^{n}}\left(\frac{\partial}{\partial z_{j}} a^{i j}\left(z, \xi_{0}\right)+b^{i}\left(z, \xi_{0}\right)\right) p^{m}(z, 0) d z, \quad m=1,2 .
$$

Из определения следует, что функция $p^{1}(0)$, а с ней и $\eta^{1}(0)$, измеримы относительно $\sigma$-алгебры $\mathscr{F} \leqslant T / 2$.

В силу условия перемешивания $\mathbf{A 3}$ имеем

$$
\left\|\mathrm{E}\left\{\eta^{1}(0) \mid \mathscr{F}_{\geqslant}\right\}\right\|_{L^{2}(\Omega)} \leqslant \rho(T / 2)\left\|\eta^{1}(0)\right\|_{L^{2}(\Omega)} \leqslant C \rho(T / 2) .
$$


Из (17) следует, что

$$
\left|p^{2}(z, 0)\right|=\left|p(z, 0)-p^{T / 2}(z, 0)\right| \leqslant c_{1} \exp (-c T / 2) .
$$

Следовательно,

$$
\left\|\mathrm{E}\left\{\eta^{2}(0) \mid \mathscr{F} \geqslant T\right\}\right\|_{L^{2}(\Omega)} \leqslant\left\|\eta^{2}(0)\right\|_{L^{2}(\Omega)} \leqslant C \exp (-c T / 2),
$$

что вместе с (29) дает требуемую оценку (28).

Согласно [18; гл. 9] при вьполнении условия $\mathbf{A 3}$ неравенство (28) обеспечивает функциональную центральную предельную теорему (ЦПТ) для процесса $\eta(-s)$, что, в свою очередь, влечет функциональную ЦПТ для $\eta(s)$. Действительно, по теореме Прохорова (см. [5]) слабая компактность в $(C[0, T])^{n}$ семейства $\left\{\varepsilon \int_{0}^{t / \varepsilon^{2}} \eta(s) d s\right\}$ является следствием слабой компактности $\left\{\varepsilon \int_{0}^{t / \varepsilon^{2}} \eta(-s) d s\right\}$ и стационарности. Сходимость конечномерных распределений процесса $\left\{\varepsilon \int_{0}^{t / \varepsilon^{2}} \eta(s) d s\right\}$ очевидньм образом эквивалентна сходимости соответствуюших конечномерных распределений $\left\{\varepsilon \int_{0}^{t / \varepsilon^{2}} \eta(-s) d s\right\}$

ЗАмЕчАниЕ. Утверждение леммы было доказано в предположении, что вьполнено последнее из неравенств в А3. Два других случая, когда имеет место равномерное или сильное перемешивание, рассматриваются аналогично.

Рассмотрим еще одну вспомогательную задачу

$$
\begin{gathered}
\frac{\partial}{\partial s} \psi^{i}(z, s)+A^{*} \psi^{i}(z, s)=-\frac{\partial}{\partial z_{j}}\left(a^{i j}\left(z, \xi_{s}\right) p(z, s)\right)-a^{i j}\left(z, \xi_{s}\right) \frac{\partial}{\partial z_{j}} p(z, s) \\
\quad+b^{i}\left(z, \xi_{s}\right) p(z, s)-\bar{b}^{i} p(z, s)-\eta^{i}(s) p(z, s), \quad(z, s) \in \mathbb{T}^{n} \times(-\infty,+\infty)
\end{gathered}
$$

здесь $A^{*}$ задан формулой (6).

Лемма 4. Задача (30) имеет стационарное әргодическое решение. В условиях нормировки

$$
\int_{\mathbb{T}^{n}} \psi(z, s) d z=0
$$

такое решение является единственным.

ДокАЗАТЕльство. Обозначим для краткости правую часть в $(30)$ через $F(z, s)$ и рассмотрим следующую последовательность задач Коши:

$$
\begin{gathered}
\frac{\partial}{\partial s} \psi^{N}(z, s)+A^{*} \psi^{N}(z, s)=\mathbf{1}_{\{N-1<s \leqslant N\}} F(z, s), \quad(z, s) \in \mathbb{T}^{n} \times(-\infty, N), \\
\left.\psi^{N}\right|_{s=N}=0 ;
\end{gathered}
$$


здесь $\mathbf{1}_{\{N-1<s \leqslant N\}}$ обозначает индикаторную функцию интервала $(N-1, N)$.

Принимая во внимание равенство $\int_{\mathbb{T}^{n}} F(z, s) d z \equiv 0$, легко проверить, что

$$
\int_{\mathbb{T}^{n}} \psi^{N}(z, s) d z=0
$$

при всех $s$; для удобства мы доопределили $\psi^{N}$ нулем при $s>N$. С помошњю (20) получаем оценку

$$
\left\|\psi^{N}\right\|_{L^{\infty}\left(\mathbb{T}^{n} \times(k, k+1)\right)} \leqslant c_{1} \exp (-c(N-k))
$$

при всех $k \leqslant N$. Суммируя ее по $N,-\infty<N<+\infty$, мы приходим к стационарному решению $\psi=\sum_{N=-\infty}^{+\infty} \psi^{N}$ задачи (30). При этом

$$
\|\psi\|_{L^{\infty}\left(\mathbb{T}^{n} \times(-\infty,+\infty)\right)} \leqslant C
$$

с некоторой неслучайной постоянной $C$. Единственность и эргодичность могут теперь быть доказаны так же, как в лемме 2.

Для того чтобы завершить доказательство компактности распределений $\left\{u^{\varepsilon}\right\}$, рассмотрим для произвольной функции $\varphi \in C_{0}^{\infty}\left(\mathbb{R}^{n}\right)$ следуюшее выражение:

$$
\left(\widetilde{u}^{\varepsilon}, \widetilde{p}^{\varepsilon} \varphi\right)+\varepsilon\left(\widetilde{u}^{\varepsilon}, \widetilde{\psi}^{\varepsilon} \nabla_{x} \varphi\right)
$$

В котором

$$
\begin{aligned}
& \widetilde{u}^{\varepsilon}(x, t)=u^{\varepsilon}\left(x-\frac{1}{\varepsilon} \bar{b} t-\frac{1}{\varepsilon} \int_{0}^{t} \eta\left(\frac{s}{\varepsilon^{2}}\right) d s, t\right) \\
& \widetilde{p}^{\varepsilon}(x, t)=p\left(\frac{x}{\varepsilon}-\varepsilon^{-2} \bar{b} t-\varepsilon^{-2} \int_{0}^{t} \eta\left(\frac{s}{\varepsilon^{2}}\right) d s, \frac{t}{\varepsilon^{2}}\right), \\
& \widetilde{\psi}^{\varepsilon}(x, t)=\psi\left(\frac{x}{\varepsilon}-\varepsilon^{-2} \bar{b} t-\varepsilon^{-2} \int_{0}^{t} \eta\left(\frac{s}{\varepsilon^{2}}\right) d s, \frac{t}{\varepsilon^{2}}\right) .
\end{aligned}
$$

Мы также будем использовать обозначения

$$
\widetilde{\varphi}=\varphi\left(x+\frac{1}{\varepsilon} \bar{b} t+\frac{1}{\varepsilon} \int_{0}^{t} \eta\left(\frac{s}{\varepsilon^{2}}\right) d s\right)
$$

и, для произвольной функции $r$,

$$
\nabla_{x}^{\varepsilon} r(x)=\left.\nabla_{z} r(z)\right|_{z=x / \varepsilon}, \quad \partial_{t}^{\varepsilon} r(t)=\left.\frac{\partial}{\partial s} r(s)\right|_{s=t / \varepsilon^{2}}
$$


С помощью уравнений (16) и (30), интегрируя по частям и вьполняя простые преобразования, найдем

$$
\begin{aligned}
& \frac{d}{d t}\left[\left(\widetilde{u}^{\varepsilon}, \widetilde{p}^{\varepsilon} \varphi\right)+\varepsilon\left(\widetilde{u}^{\varepsilon}, \widetilde{\psi}^{\varepsilon} \nabla_{x} \varphi\right)\right]=\frac{d}{d t}\left[\left(u^{\varepsilon}, p^{\varepsilon} \widetilde{\varphi}\right)+\varepsilon\left(u^{\varepsilon}, \psi^{\varepsilon} \nabla_{x} \widetilde{\varphi}\right)\right] \\
&=\left(A^{\varepsilon} u^{\varepsilon}, p^{\varepsilon} \widetilde{\varphi}\right)+\left(u^{\varepsilon}, \widetilde{\varphi} \frac{\partial}{\partial t} p^{\varepsilon}\right)+\frac{1}{\varepsilon}\left(\bar{b}+\eta\left(t / \varepsilon^{2}\right)\right) \cdot\left(u^{\varepsilon}, p^{\varepsilon} \nabla_{x} \widetilde{\varphi}\right) \\
&+\varepsilon\left(A^{\varepsilon} u^{\varepsilon}, \psi^{\varepsilon} \nabla_{x} \widetilde{\varphi}\right)+\varepsilon\left(u^{\varepsilon}, \nabla_{x} \widetilde{\varphi} \frac{\partial}{\partial t} \psi^{\varepsilon}\right)+\left(\bar{b}+\eta\left(t / \varepsilon^{2}\right)\right) \cdot\left(u^{\varepsilon}, \psi^{\varepsilon} \nabla_{x} \nabla_{x} \widetilde{\varphi}\right) \\
&=\left(u^{\varepsilon}, p^{\varepsilon} a^{\varepsilon} \nabla_{x} \nabla_{x} \widetilde{\varphi}\right)+\left(u^{\varepsilon} \nabla_{x} \nabla_{x} \widetilde{\varphi},\left[\nabla_{x}^{\varepsilon}(a \psi)+a^{\varepsilon} \nabla_{x}^{\varepsilon} \psi-b^{\varepsilon} \psi^{\varepsilon}+\bar{b} \psi^{\varepsilon}+\eta^{\varepsilon} \psi^{\varepsilon}\right]\right) \\
&+\varepsilon\left(u^{\varepsilon} \psi^{\varepsilon}, a^{\varepsilon} \nabla_{x} \nabla_{x} \nabla_{x} \widetilde{\varphi}\right) .
\end{aligned}
$$

С учетом (3) отсюда следует неравенство

$$
\left|\left(\widetilde{u}^{\varepsilon}(t), \widetilde{p}^{\varepsilon}(t) \varphi\right)-\left(\widetilde{u}^{\varepsilon}(s), \widetilde{p}^{\varepsilon}(s) \varphi\right)\right| \leqslant c|t-s|\|\varphi\|_{C^{3}} .
$$

Приближая, если необходимо, начальное условие $u_{0} \in L^{2}\left(\mathbb{R}^{n}\right)$ в задаче (1) последовательностью функций $u_{0}^{m} \in C_{0}^{\infty}\left(\mathbb{R}^{n}\right)$ и используя $(3)$, мы всегда можем предполагать, что $u_{0} \in C_{0}^{\infty}\left(\mathbb{R}^{n}\right)$.

Тогда ввиду (34) и леммы 1 имеем

$$
\left|\left(\widetilde{u}^{\varepsilon}(t), \varphi\right)-\left(\widetilde{u}^{\varepsilon}(s), \varphi\right)\right| \leqslant c|t-s|\|\varphi\|_{C^{3}}+c \kappa_{1}(\varepsilon)\|\varphi\|_{C^{1}} .
$$

Обозначая

$$
\widehat{u}^{\varepsilon}(x, t)=u^{\varepsilon}\left(x-\varepsilon^{-1} \bar{b} t, t\right),
$$

приходим к следуюшему соотношению:

$$
\begin{aligned}
\left|\left(\widehat{u}^{\varepsilon}(t), \varphi\right)-\left(\widehat{u}^{\varepsilon}(s), \varphi\right)\right| & \left|\left(\widetilde{u}^{\varepsilon}(t), \varphi\left(\cdot-\frac{1}{\varepsilon} \int_{0}^{t} \eta\left(\tau / \varepsilon^{2}\right) d \tau\right)\right)-\left(\widetilde{u}^{\varepsilon}(s), \varphi\left(-\frac{1}{\varepsilon} \int_{0}^{s} \eta\left(\tau / \varepsilon^{2}\right) d \tau\right)\right)\right| \\
\leqslant & \left|\left(\widetilde{u}^{\varepsilon}(t), \varphi\left(\cdot-\frac{1}{\varepsilon} \int_{0}^{s} \eta\left(\tau / \varepsilon^{2}\right) d \tau\right)\right)-\left(\widetilde{u}^{\varepsilon}(s), \varphi\left(\cdot-\frac{1}{\varepsilon} \int_{0}^{s} \eta\left(\tau / \varepsilon^{2}\right) d \tau\right)\right)\right| \\
& +\left|\left(\widetilde{u}^{\varepsilon}(t), \varphi\left(-\frac{1}{\varepsilon} \int_{0}^{t} \eta\left(\tau / \varepsilon^{2}\right) d \tau\right)-\varphi\left(\cdot-\frac{1}{\varepsilon} \int_{0}^{s} \eta\left(\tau / \varepsilon^{2}\right) d \tau\right)\right)\right| \\
\leqslant & c|t-s|\|\varphi\|_{C^{3}}+c \kappa(\varepsilon)\|\varphi\|_{C^{1}}+c\|\varphi\|_{C^{1}}\left|\frac{1}{\varepsilon} \int_{s}^{t} \eta\left(\tau / \varepsilon^{2}\right) d \tau\right| .
\end{aligned}
$$

Для произвольной $\varphi \in C_{0}^{\infty}\left(\mathbb{R}^{n}\right)$ первые два члена в правой части последней формулы стремятся к нулю при $\varepsilon \rightarrow 0$ и $|t-s| \rightarrow 0$, равномерно по $t, s$ и $\omega \in \Omega$. По лемме 3 интеграл $\frac{1}{\varepsilon} \int_{0}^{t} \eta\left(\tau / \varepsilon^{2}\right) d \tau$ удовлетворяет функциональной ЦПТ в $(C[0, T])^{n}$ на любом интервале $[0, T]$. Поэтому, применяя дважды теорему Прохорова, мы убеждаемся в слабой компактности семейства распределений $\left(\widetilde{u}^{\varepsilon}, \varphi\right)$ в пространстве $C(0, T)$. 


\section{§4. Предельный переход}

Цель этого параграфа - показать, что семейство функций $\left\{\widetilde{u}^{\varepsilon}\right\}$ сходится почти наверное к решению задачи Коши для предельного параболического детерминированного уравнения с постоянными коэффициентами, а затем получить основные результаты о сходимости по распределению для семейства решений задачи (1). Сначала мы обоснуем сходимость в пространстве $V$, введенном в $\S 2$, а затем покажем, что результат о сходимости остается справедливым в более сильной топологии.

Поскольку компактность семейства $\left\{\widetilde{u}^{\varepsilon}\right\}$ уже доказана, то для того чтобы найти предельное распределение, достаточно перейти к пределу в выражениях вида $\left(\widetilde{u}^{\varepsilon}, \varphi\right)$ с произвольной $\varphi \in C_{0}^{\infty}$.

Согласно (33) и лемме 1 получаем

$$
\begin{aligned}
\left(\widetilde{u}^{\varepsilon}(t), \varphi\right)-\left(u_{0}, \varphi\right) \\
=\left(\widetilde{u}^{\varepsilon}(t), p^{\varepsilon}(t) \varphi\right)+\varepsilon\left(\widetilde{u}^{\varepsilon}(t), \psi^{\varepsilon}(t) \nabla_{x} \varphi\right)-\left(u_{0}, p^{\varepsilon}(0) \varphi\right)-\varepsilon\left(u_{0}, \psi^{\varepsilon}(0) \nabla_{x} \varphi\right)+O(\varepsilon) \\
=\int_{0}^{t}\left(u^{\varepsilon}(s), a^{\varepsilon} p^{\varepsilon}(s) \nabla_{x} \nabla_{x} \widetilde{\varphi}\right) d s+\int_{0}^{t}\left(u^{\varepsilon}(s), a^{\varepsilon} \nabla_{x}^{\varepsilon} \psi^{\varepsilon}(s) \nabla_{x} \nabla_{x} \widetilde{\varphi}\right) d s \\
\quad+\int_{0}^{t}\left(u^{\varepsilon}(s), \nabla_{x}^{\varepsilon}\left(a^{\varepsilon} \psi^{\varepsilon}(s)\right) \nabla_{x} \nabla_{x} \widetilde{\varphi}\right) d s-\int_{0}^{t}\left(u^{\varepsilon}(s), b^{\varepsilon} \psi^{\varepsilon}(s) \nabla_{x} \nabla_{x} \widetilde{\varphi}\right) d s \\
\quad+\int_{0}^{t}\left(u^{\varepsilon}(s), \bar{b} \psi^{\varepsilon}(s) \nabla_{x} \nabla_{x} \widetilde{\varphi}\right) d s+\int_{0}^{t}\left(u^{\varepsilon}(s), \eta^{\varepsilon}(s) \psi^{\varepsilon}(s) \nabla_{x} \nabla_{x} \widetilde{\varphi}\right) d s+O(\varepsilon) .
\end{aligned}
$$

Перейти к пределу в этом выражении нам поможет следующая лемма.

Лемма 5. Пусть $\zeta(z, s)$ - стационарныц әргодический прочесс со значениями в $L^{2}\left(\mathbb{T}^{n}\right)\left(C\left(\mathbb{T}^{n}\right)\right)$ maкой, что

$$
\|\zeta\|_{L^{2}\left(\mathbb{T}^{n} \times(0,1)\right)} \leqslant C
$$

с неслучайной константой $C$. Тогда для всякой $C_{0}^{\infty}$-функции $\varphi$ п.н. выполнено соотношение

$$
\lim _{\varepsilon \rightarrow 0} \sup _{t \leqslant T}\left|\int_{0}^{t}\left(u^{\varepsilon}(s), \zeta^{\varepsilon}(s) \widetilde{\varphi}\right) d s-\overline{\langle\zeta\rangle} \int_{0}^{t}\left(u^{\varepsilon}(s), \widetilde{\varphi}\right) d s\right|=0
$$

где

$$
\overline{\langle\zeta\rangle}=\mathrm{E} \int_{\mathbb{T}^{n}} \zeta(z, s) d z
$$

и $\widetilde{\varphi}$ определена в (32).

ДокАЗАТЕЛЬСТво. Без ограничения обшности будем предполагать, что $\overline{\langle\zeta\rangle}=0$, и обозначим $\mu(s)=\int_{\mathbb{T}^{n}} \zeta(z, s) d z$. Тогда по лемме 1 имеем

$$
\sup _{t \leqslant T}\left|\int_{0}^{t}\left[\left(u^{\varepsilon}(\tau), \zeta^{\varepsilon}(\tau) \widetilde{\varphi}\right)-\mu\left(\frac{\tau}{\varepsilon^{2}}\right)\left(u^{\varepsilon}(\tau), \widetilde{\varphi}\right)\right] d \tau\right| \leqslant C \kappa_{1}(\varepsilon)
$$


с неслучайной постоянной $C$. Таким образом, достаточно показать, что

$$
\lim _{\varepsilon \rightarrow 0} \sup _{t \leqslant T}\left|\int_{0}^{t} \mu\left(\frac{\tau}{\varepsilon^{2}}\right)\left(u^{\varepsilon}(\tau), \widetilde{\varphi}\right) d \tau\right|=0
$$

Из (35) по теореме Арцела следует, что для каждой $\varphi \in C_{0}^{\infty}\left(\mathbb{R}^{n}\right)$ найдется такое компактное множество $K \subset C[0, T]$, что $\left(\widetilde{u}^{\varepsilon}(\cdot), \varphi\right) \in K$ п.н. при всех $\varepsilon>0$. Дальнейшее доказательство следует стандартной схеме. Мы строим конечную $\delta$-сеть, состоящую из ступенчатых функций, и применяем теорему Биркгофа, чтобы доказать предельное соотношение

$$
\lim _{\varepsilon \rightarrow 0}\left|\int_{0}^{t} \mu\left(\frac{\tau}{\varepsilon^{2}}\right)\left(u^{\varepsilon}(\tau), \widetilde{\varphi}\right) d \tau\right|=0
$$

для каждого $t \leqslant T$. В сочетании с простой оценкой

$$
\left|\int_{s}^{t} \mu\left(\frac{\tau}{\varepsilon^{2}}\right)\left(u^{\varepsilon}(\tau), \widetilde{\varphi}\right) d \tau\right| \leqslant c\left\|\mu\left(\frac{\cdot}{\varepsilon^{2}}\right)\right\|_{L^{2}(0, T)}|t-s|^{1 / 2}
$$

это дает (40), что завершает доказательство леммы.

Будем обозначать $\bar{a}^{i j}$ “усредненную” матрицу, заданную формулой

$$
\bar{a}^{i j}=\mathrm{E} \int_{\mathbb{T}^{n}}\left[a^{i j}\left(z, \xi_{s}\right) p(z, s)+a^{i k}\left(z, \xi_{s}\right) \frac{\partial}{\partial z_{k}} \psi^{j}(z, s)-b^{i}\left(z, \xi_{s}\right) \psi^{j}(z, s)\right] d z .
$$

Это определение естественно, поскольку члены в правой части (38), не принимаюшие участия в определении $\bar{a}^{i j}$, удовлетворяют равенству

$$
\int_{\mathbb{T}^{n}}\left(\frac{\partial}{\partial z_{k}}\left(a^{i k}\left(z, \xi_{s}\right) \psi^{j}(z, s)\right)+\bar{b}^{i} \psi^{j}(z, s)+\eta^{i}(s) \psi^{j}(z, s)\right) d z=0
$$

По лемме 5 с учетом (38) и последнего равенства получим

$$
\lim _{\varepsilon \rightarrow 0} \sup _{t \leqslant T}\left|\left(\widetilde{u}^{\varepsilon}(t), \varphi\right)-\left(u_{0}, \varphi\right)-\int_{0}^{t}\left(\widetilde{u}^{\varepsilon}(s), \bar{a} \nabla_{x} \nabla_{x} \varphi\right) d s\right|=0
$$

п.н. для каждой $\varphi \in C_{0}^{\infty}$. Таким образом, для типичной реализации $\xi$. любая предельная точка семейства $\left\{\widetilde{u}^{\varepsilon}\right\}$ удовлетворяет уравнению

$$
\frac{\partial}{\partial t} u^{0}-\bar{A} u^{0}=0,\left.\quad u^{0}\right|_{t=0}=u_{0}
$$

с оператором $\bar{A}=\bar{a}^{i j} \frac{\partial}{\partial x_{i}} \frac{\partial}{\partial x_{j}}$. Решение этой задачи единственно, поэтому семейство $\left\{\widetilde{u}^{\varepsilon}\right\}$ п.н. сходится в $V$ к функции $u^{0}$ при $\varepsilon \rightarrow 0$. 
В действительности этот результат о сходимости может быть усилен. Мы покажем, что можно так выбрать стационарный эргодический процесс $\chi(z, s)$ со значениями в $C\left(\mathbb{T}^{n}\right)$, что функшия

$$
\begin{aligned}
v^{\varepsilon}(x, t) \equiv & u^{\varepsilon}(x, t)-u^{0}\left(x+\bar{b} \frac{t}{\varepsilon}+\frac{1}{\varepsilon} \int_{0}^{t} \eta\left(\frac{\tau}{\varepsilon^{2}}\right) d \tau, t\right) \\
& -\varepsilon \nabla_{x} u^{0}\left(x+\bar{b} \frac{t}{\varepsilon}+\frac{1}{\varepsilon} \int_{0}^{t} \eta\left(\frac{\tau}{\varepsilon^{2}}\right) d \tau, t\right) \chi\left(\frac{x}{\varepsilon}, \frac{t}{\varepsilon^{2}}\right)
\end{aligned}
$$

сходится к нулю п.н. в норме пространства $L^{\infty}\left(\mathbb{R}^{n} \times[0, T]\right) \cap L^{2}\left(0, T ; H^{1}\left(\mathbb{R}^{n}\right)\right)$. Для этого подставим выражение (43) в исходное уравнение, сгруппируем члены с одинаковыми степенями $\varepsilon$ и приравняем к нулю каждое из полученных выражений. Первое уравнение в этой цепочке порождается членами порядка $\varepsilon^{-1}$, оно имеет вид

$$
\begin{aligned}
& \left.\left(\frac{\partial}{\partial s} \chi(z, s)-A \chi(z, s)\right)\right|_{z=\frac{x}{\varepsilon}, s=\frac{t}{\varepsilon^{2}}} \nabla_{x} u_{\varepsilon}^{0}(x, t) \\
& \quad=\left.\left[\nabla_{z} a\left(z, \xi_{s}\right)+b\left(z, \xi_{s}\right)-\bar{b}-\eta(s)\right]\right|_{z=\frac{x}{\varepsilon}, s=\frac{t}{\varepsilon^{2}}} \nabla_{x} u_{\varepsilon}^{0}(x, t) ;
\end{aligned}
$$

здесь и далее мы обозначаем

$$
u_{\varepsilon}^{0}(x, t)=u^{0}\left(x+\bar{b} \frac{t}{\varepsilon}+\frac{1}{\varepsilon} \int_{0}^{t} \eta\left(\frac{\tau}{\varepsilon^{2}}\right) d \tau, t\right) .
$$

Поэтому мы хотим выбрать функщию $\chi(z, s)$ как решение уравнения

$$
\frac{\partial}{\partial s} \chi^{j}(z, s)-A \chi^{j}(z, s)=\nabla_{z_{i}} a^{i j}\left(z, \xi_{s}\right)+b^{j}\left(z, \xi_{s}\right)-\bar{b}^{j}-\eta^{j}(s) .
$$

Изучим подробнее это уравнение. Умножив его на $p(z, s)$, проинтегрировав в цилиндpe $\mathbb{T}^{n} \times\left[s_{1}, s_{2}\right]$ и вспомнив определения $p(z, s), \bar{b}$ и $\eta(s)$, легко получить равенство

$$
\int_{\mathbb{T}^{n}} \chi(z, s) p(z, s) d z=\text { const. }
$$

Далее, при помоши тех же рассуждений, что при доказательстве леммы 4, можно показать, что уравнение (44) имеет стационарное решение $\chi(z, s)$, единственное с точностью до аддитивной константы. Более того, $\chi(\cdot, s)$ является эргодическим процессом со значениями в $C\left(\mathbb{T}^{n}\right)$. Для определенности положим $\int_{\mathbb{T}^{n}} \chi(z, s) p(z, s) d z=0$.

При указанном выборе $\chi$ имеем

$$
\begin{aligned}
\left(\frac{\partial}{\partial t}-A^{\varepsilon}\right) v^{\varepsilon}= & \left(-\frac{\partial}{\partial t}+\check{a}^{i j} \frac{\partial}{\partial x_{i}} \frac{\partial}{\partial x_{j}}\right) u^{0}+\left(a^{\varepsilon}+\nabla_{x}^{\varepsilon}\left(a^{\varepsilon} \chi^{\varepsilon}\right)+a^{\varepsilon} \nabla_{x}^{\varepsilon} \chi^{\varepsilon}\right. \\
& \left.+b^{\varepsilon} \chi^{\varepsilon}-\bar{b} \chi^{\varepsilon}-\eta\left(\frac{t}{\varepsilon^{2}}\right) \chi^{\varepsilon}-\check{a}\right) \nabla_{x} \nabla_{x} u^{0}+O(\varepsilon),
\end{aligned}
$$


где $O(\varepsilon)$ сходится к нулю в $L^{\infty}\left(0, T ; H^{-1}\left(\mathbb{R}^{n}\right)\right)$-норме равномерно по $\omega \in \Omega$ и

$$
\begin{aligned}
\check{a}^{i j}= & \mathrm{E} \int_{\mathbb{T}^{n}}\left(a^{i j}\left(z, \xi_{s}\right)+\nabla_{z_{k}}\left(a^{i k}\left(z, \xi_{s}\right) \chi^{j}(z, s)\right)\right. \\
& \left.+a^{i k}\left(z, \xi_{s}\right) \nabla_{z_{k}} \chi^{j}(z, s)+b^{i}\left(z, \xi_{s}\right) \chi^{j}(z, s)\right) p(z, s) d z .
\end{aligned}
$$

Для краткости введем обозначения

$$
\begin{aligned}
\check{a}_{\mathrm{aux}}^{\varepsilon}(x, t)=\left\{\check{a}_{\mathrm{aux}}^{\varepsilon, i j}(x, t)\right\}= & a\left(\frac{x}{\varepsilon}, \xi_{t / \varepsilon^{2}}\right)+\nabla_{x}^{\varepsilon}\left(a\left(\frac{x}{\varepsilon}, \xi_{t / \varepsilon^{2}}\right) \chi\left(\frac{x}{\varepsilon}, \frac{t}{\varepsilon^{2}}\right)\right) \\
& +a\left(\frac{x}{\varepsilon}, \xi_{t / \varepsilon^{2}}\right) \nabla_{x}^{\varepsilon} \chi\left(\frac{x}{\varepsilon}, \frac{t}{\varepsilon^{2}}\right) \\
& +b\left(\frac{x}{\varepsilon}, \xi_{t / \varepsilon^{2}}\right) \chi\left(\frac{x}{\varepsilon}, \frac{t}{\varepsilon^{2}}\right)-\left(\bar{b}+\eta\left(\frac{t}{\varepsilon^{2}}\right)\right) \chi\left(\frac{x}{\varepsilon}, \frac{t}{\varepsilon^{2}}\right)
\end{aligned}
$$

и

$$
\begin{aligned}
\left\langle\check{a}_{\mathrm{aux}}\right\rangle(s)= & \int_{\mathbb{T}^{n}}\left\{a^{i j}\left(z, \xi_{s}\right)+\nabla_{z_{k}}\left(a^{i k}\left(z, \xi_{s}\right) \chi^{j}(z, s)\right)\right. \\
& \left.+a^{i k}\left(z, \xi_{s}\right) \nabla_{z_{k}} \chi^{j}(z, s)+b^{i}\left(z, \xi_{s}\right) \chi^{j}(z, s)\right\} p(z, s) d z .
\end{aligned}
$$

В дальнейшем будет ясно, что $\check{a}=\bar{a}$. Следовательно, первый член в правой части (45) обрашается в нуль. Для получения энергетической оценки умножим (45) на $p^{\varepsilon} v^{\varepsilon}$ и проинтегрируем результат на множестве $\mathbb{R}^{n} \times(0, t)$. После простых преобразований это дает

$$
\begin{aligned}
& \int_{\mathbb{R}^{n}}\left(v^{\varepsilon}(x, t)\right)^{2} p\left(\frac{x}{\varepsilon}, \frac{t}{\varepsilon^{2}}\right) d x+\int_{0}^{t} \int_{\mathbb{R}^{n}} p\left(\frac{x}{\varepsilon}, \frac{s}{\varepsilon^{2}}\right) a^{i j}\left(\frac{x}{\varepsilon}, \xi_{\frac{s}{\varepsilon^{2}}}\right) \frac{\partial}{\partial x_{i}} v^{\varepsilon}(x, s) \frac{\partial}{\partial x_{j}} v^{\varepsilon}(x, s) d x d s \\
&= \varepsilon^{2} \int_{\mathbb{R}^{n}} p\left(\frac{x}{\varepsilon}, 0\right)\left(\chi^{i}\left(\frac{x}{\varepsilon}, 0\right) \frac{\partial}{\partial x_{i}} u_{0}(x)\right)^{2} d x \\
&+\int_{0}^{t} \int_{\mathbb{R}^{n}}\left[\check{a}_{\text {aux }}^{\varepsilon, i j}(x, s)-\check{a}^{i j}\right] p\left(\frac{x}{\varepsilon}, \frac{s}{\varepsilon^{2}}\right) \frac{\partial}{\partial x_{i}} \frac{\partial}{\partial x_{j}} u_{\varepsilon}^{0}(x, s) v^{\varepsilon}(x, s) d x d s \\
&= \varepsilon^{2} \int_{\mathbb{R}^{n}} p\left(\frac{x}{\varepsilon}, 0\right)\left(\chi^{i}\left(\frac{x}{\varepsilon}, 0\right) \frac{\partial}{\partial x_{i}} u_{0}(x)\right)^{2} d x \\
&+\int_{0}^{t} \int_{\mathbb{R}^{n}}\left[\check{a}_{\text {aux }}^{\varepsilon, i j}(x, s)-\left\langle\check{a}_{\text {aux }}\right\rangle^{i j}\left(\frac{s}{\varepsilon^{2}}\right)\right] p\left(\frac{x}{\varepsilon}, \frac{s}{\varepsilon^{2}}\right) \frac{\partial}{\partial x_{i}} \frac{\partial}{\partial x_{j}} u_{\varepsilon}^{0}(x, s) v^{\varepsilon}(x, s) d x d s \\
&+\int_{0}^{t} \int_{\mathbb{R}^{n}}\left[\left\langle\check{a}_{\text {aux }}\right\rangle^{i j}\left(\frac{s}{\varepsilon^{2}}\right)-\check{a}^{i j}\right] p\left(\frac{x}{\varepsilon}, \frac{s}{\varepsilon^{2}}\right) \frac{\partial}{\partial x_{i}} \frac{\partial}{\partial x_{j}} u_{\varepsilon}^{0}(x, s) v^{\varepsilon}(x, s) d x d s .
\end{aligned}
$$

Второй интеграл в правой части этой формулы допускает оценку

$$
\begin{gathered}
\left|\int_{0}^{t} \int_{\mathbb{R}^{n}}\left[\check{a}_{\text {aux }}^{\varepsilon, i j}(x, s)-\left\langle\check{a}_{\text {aux }}\right\rangle^{i j}\left(\frac{s}{\varepsilon^{2}}\right)\right] p\left(\frac{x}{\varepsilon}, \frac{s}{\varepsilon^{2}}\right) \frac{\partial}{\partial x_{i}} \frac{\partial}{\partial x_{j}} u_{\varepsilon}^{0}(x, s) v^{\varepsilon}(x, s) d x d s\right| \\
\quad=\varepsilon\left|\int_{0}^{t} \int_{\mathbb{R}^{n}} \Psi^{i j, k}\left(\frac{x}{\varepsilon}, \frac{s}{\varepsilon^{2}}\right) \frac{\partial}{\partial x_{k}}\left(\frac{\partial}{\partial x_{i}} \frac{\partial}{\partial x_{j}} u_{\varepsilon}^{0}(x, s) v^{\varepsilon}(x, s)\right) d x d s\right| \leqslant c \varepsilon
\end{gathered}
$$


с неслучайной постоянной $c$; здесь $\Psi^{i j, k}(z, s)$ выбраны так, чтобы вьполнялось равенство

$$
\operatorname{div} \Psi^{i j}(z, s)=\left(\check{a}_{\text {aux }}^{\varepsilon, i j}(z, s)-\left\langle\check{a}_{\text {aux }}\right\rangle^{i j}\right) p(z, s) .
$$

Оценим последний член в (46). Из определения $v^{\varepsilon}(x, t)$ легко следует, что

$v^{\varepsilon}(x, t)=u^{\varepsilon}(x, t)-u^{0}\left(x-\bar{b} \frac{t}{\varepsilon}-\frac{1}{\varepsilon} \int_{0}^{t} \eta\left(\frac{\tau}{\varepsilon^{2}}\right) d \tau, t\right)+O(\varepsilon)=u^{\varepsilon}(x, t)-u_{\varepsilon}^{0}(x, t)+O(\varepsilon)$

равномерно по $x, t$ и $\omega$. Далее, согласно (35) семейство

$$
\left(u^{\varepsilon}(t)-u_{\varepsilon}^{0}(t), \nabla_{x} \nabla_{x} u_{\varepsilon}^{0}(t)\right)=\left(\widetilde{u}^{\varepsilon}(t)-u^{0}(t), \nabla_{x} \nabla_{x} u^{0}(t)\right)
$$

компактно в $(C[0, T])^{n^{2}}$, поэтому по теореме Биркгофа п.н. выполняется предельное соотношение

$$
\lim _{\varepsilon \rightarrow 0} \sup _{t \leqslant T}\left|\int_{0}^{t} \int_{\mathbb{R}^{n}}\left[\left\langle\check{a}_{\text {aux }}\right\rangle^{i j}\left(\frac{s}{\varepsilon^{2}}\right)-\check{a}^{i j}\right] p\left(\frac{x}{\varepsilon}, \frac{s}{\varepsilon^{2}}\right) \frac{\partial}{\partial x_{i}} \frac{\partial}{\partial x_{j}} u_{\varepsilon}^{0}(x, s) v^{\varepsilon}(x, s) d x d s\right|=0 .
$$

Мы приходим к следуюшей лемме.

Лемма 6. При $\varepsilon \rightarrow 0$ разность $\left(u^{\varepsilon}-u_{\varepsilon}^{0}\right)$, а с ней $и\left(\widetilde{u}^{\varepsilon}-u^{0}\right)$, сходится п.н. $к$ нулю в норме пространства $L^{\infty}\left(0, T ; L^{2}\left(\mathbb{R}^{n}\right)\right)$.

Переходим теперь к основным результатам работы. Через $\widehat{Q}^{\varepsilon}$ обозначим распределение функции $\widehat{u}^{\varepsilon}(x, t)=u^{\varepsilon}\left(x-\varepsilon^{-1} \bar{b} t, t\right)$ в $V$, причем вектор $\bar{b}$ задан формулой (25). Напомним также, что матрица $\left\{\bar{a}^{i j}\right\}$ определена в (41).

Теорема 1. Пусть $\left\{u^{\varepsilon}\right\}$-решение задачи (1); предположим, что $u_{0} \in L^{2}\left(\mathbb{R}^{n}\right)$. Тогда меры $\widehat{Q}^{\varepsilon}$ сходятся слабо в $V$ при $\varepsilon \rightarrow 0$ к единственному решению следующего стохастического уравнения в частных производных:

$$
\begin{gathered}
d u(t)=\left(\bar{a}^{i j}+\frac{1}{2}\left(\Lambda^{2}\right)^{i j}\right) \frac{\partial}{\partial x_{i}} \frac{\partial}{\partial x_{j}} u(t) d t+\Lambda \nabla_{x} u(t) d w_{t}, \\
\left.u\right|_{t=0}=u_{0}
\end{gathered}
$$

с матричей $\Lambda$, заданной формулой

$$
\left(\Lambda^{2}\right)^{i j}=\int_{0}^{\infty} \mathrm{E}\left(\eta^{i}(0) \eta^{j}(s)+\eta^{j}(0) \eta^{i}(s)\right) d s
$$

и со стандартным $n$-мерныц винеровским процессом $w_{t}$. 
ДокАЗАТЕльство. С помошњю формулы Ито легко проверить, что функция $u^{0}\left(x-\Lambda w_{t}, t\right)$ есть решение задачи (47). Согласно [8] эта задача корректна и имеет единственное решение.

Преобразуем функцию $u^{\varepsilon}\left(x-\varepsilon^{-1} \bar{b} t, t\right)$ к следующему виду:

$$
\begin{aligned}
u^{\varepsilon}\left(x-\varepsilon^{-1} \bar{b} t, t\right)= & u^{0}\left(x+\frac{1}{\varepsilon} \int_{0}^{t} \eta\left(\frac{s}{\varepsilon^{2}}\right) d s, t\right) \\
& +u^{\varepsilon}\left(x-\varepsilon^{-1} \bar{b} t, t\right)-u^{0}\left(x+\frac{1}{\varepsilon} \int_{0}^{t} \eta\left(\frac{s}{\varepsilon^{2}}\right) d s, t\right)
\end{aligned}
$$

Из леммы 6 следует, что $L^{\infty}\left(0, T ; L^{2}\left(\mathbb{R}^{n}\right)\right)$-норма разности второго и третьего членов в правой части стремится к нулю п.н. при $\varepsilon \rightarrow 0$. Поэтому для каждой $\varphi \in C_{0}^{\infty}$ семейства

$$
\left(u^{\varepsilon}\left(x-\varepsilon^{-1} \bar{b} t, t\right), \varphi\right) \text { и }\left(u^{0}\left(x+\frac{1}{\varepsilon} \int_{0}^{t} \eta\left(\frac{s}{\varepsilon^{2}}\right) d s, t\right), \varphi\right)
$$

должны иметь один и тот же предел по распределению в $C(0, T)$.

Отображение $F_{\varphi}:(C(0, T))^{n} \rightarrow C(0, T)$, заданное равенством

$$
F_{\varphi}(\theta(\cdot))=\left(u^{0}(\cdot+\theta(t), t), \varphi\right)
$$

непрерьвно, поэтому сходимость по распределению в $(C(0, T))^{n}$ функций

$$
\left\{\frac{1}{\varepsilon} \int_{0}^{t} \eta\left(\frac{s}{\varepsilon^{2}}\right) d s\right\}
$$

к $\Lambda w_{t}$ влечет сходимость по распределению функций

$$
\left(u^{0}\left(x+\frac{1}{\varepsilon} \int_{0}^{t} \eta\left(\frac{s}{\varepsilon^{2}}\right) d s, t\right), \varphi\right)
$$

к $\left(u^{0}\left(x+\Lambda w_{t}, t\right), \varphi\right)$, и теорема доказана.

Топология пространства $V$ весьма слабая. Следующий результат показьвает, что сходимость остается справедливой в более сильной топологии.

Теорема 2. Семейство $\left\{\widehat{u}^{\varepsilon}\right\}$ сходится по распределению в функииональном пространстве $V^{1}=L^{\infty}\left(0, T ; L^{2}\left(\mathbb{R}^{n}\right)\right)$ с сильной топологией (т.е. топологией сходимости по норме).

ДокАЗАТЕльство. Мы уже доказали, что разность

$$
u^{\varepsilon}\left(x-\varepsilon^{-1} \bar{b} t, t\right)-u^{0}\left(x+\frac{1}{\varepsilon} \int_{0}^{t} \eta\left(\frac{s}{\varepsilon^{2}}\right) d s, t\right)
$$

сходится к нулю в пространстве $V^{1}$, т.е. в $L^{\infty}\left(0, T ; L^{2}\left(\mathbb{R}^{n}\right)\right)$-норме. По теореме Прохорова и в силу леммы 3 для каждого $\delta>0$ существует компактное подмножество $K \in(C(0, T))^{n}$ такое, что

$$
\sup _{\varepsilon>0} \mathrm{P}\left\{\frac{1}{\varepsilon} \int_{0}^{t} \eta\left(\frac{s}{\varepsilon^{2}}\right) d s \notin K\right\}<\delta .
$$


Отображение $\Phi:(C(0, T))^{n} \rightarrow V^{1}$, заданное равенством

$$
\Phi(\theta(\cdot))=u^{0}(x+\theta(t), t)
$$

непрерьвно, поэтому для каждого $\delta>0$ найдется компактное множество $K^{1}$ в $V^{1}$ такое, что

$$
\sup _{\varepsilon>0} \mathrm{P}\left\{u^{0}\left(x+\frac{1}{\varepsilon} \int_{0}^{t} \eta\left(\frac{s}{\varepsilon^{2}}\right) d s, t\right) \notin K^{1}\right\}<\delta .
$$

Благодаря лемме 6 отсюда следует совпадение пределов по распределению в $V^{1}$ для семейств

$$
\left\{u^{\varepsilon}\left(x-\varepsilon^{-1} \bar{b} t, t\right)\right\} \text { и }\left\{u^{0}\left(x+\frac{1}{\varepsilon} \int_{0}^{t} \eta\left(\frac{s}{\varepsilon^{2}}\right) d s, t\right)\right\} .
$$

Сходимость по распределению выражений $u^{0}\left(x+\frac{1}{\varepsilon} \int_{0}^{t} \eta\left(\frac{s}{\varepsilon^{2}}\right) d s, t\right)$ к $u^{0}\left(x+\Lambda w_{t}, t\right)$ следует из леммы 3 и непрерьвности отображения $\Phi$, построенного выше.

\section{§5. Операторы с диффузионными коэффициентами}

В этом параграфе мы предполагаем, что процесс $\xi$., участвующий в определении коэффициентов уравнения (1), является диффузионньм. В этом частном случае все коэффициенты эффективного уравнения (47) могут быть найдены в терминах решений вспомогательных детерминированных уравнений в частных производных. Кроме того, различные достаточные условия, обеспечиваюшие свойства перемешивания $\mathbf{A 3}$ процесса $\xi$., могут быть сформулированы в терминах коэффициентов генератора $\xi$. .

Напомним обозначение $\zeta_{s}=\xi_{-s}$ и рассмотрим диффузионный процесс $\left(\widetilde{X}_{s}, \zeta_{s}\right)$ со значениями в $\mathbb{T}^{n} \times \mathbb{R}^{d}$, соответствуюший оператору

$$
A+\widetilde{L}=\frac{\partial}{\partial z_{i}} a_{i j}(z, y) \frac{\partial}{\partial z_{j}}+b_{i}(z, y) \frac{\partial}{\partial z_{i}}+\widetilde{q}_{k l}(y) \frac{\partial}{\partial y_{k}} \frac{\partial}{\partial y_{l}}+\widetilde{B}_{k}(y) \frac{\partial}{\partial y_{k}} .
$$

Согласно $[20]$ и $[7]$ в предположениях $\mathbf{A 1}, \mathbf{A} \mathbf{2}$ и $\mathbf{A} \mathbf{3}^{\prime}$ процесс $\left(\widetilde{X}_{s}, \zeta_{s}\right)$ имеет единственную инвариантную меру, и плотность этой меры удовлетворяет уравнению

$$
\left(A^{*}+\widetilde{L}^{*}\right) \widetilde{\rho}(z, y)=0, \quad \int_{\mathbb{T}^{n}} \int_{\mathbb{R}^{d}} \widetilde{\rho}(z, y) d y d z=1 .
$$

Более того, $\widetilde{\rho}(z, y)$ убьвает при $|y| \rightarrow \infty$ быстрее, чем любая отрицательная степень $|y|$.

Далее, если $f(z, y)$ - функция полиномиального роста по $y$, то уравнение

$$
(A+\widetilde{L}) \widetilde{\chi}(z, y)=f(z, y)
$$

разрешимо тогда и только тогда, когда

$$
\int_{\mathbb{T}^{n}} \int_{\mathbb{R}^{d}} f(z, y) \widetilde{\rho}(z, y) d y d z=0 ;
$$


при этом решение также имеет не более чем полиномиальный рост по $y$.

Мы предполагаем в дальнейшем, что $\left(\widetilde{X}_{s}, \zeta_{s}\right)$ - это стационарный процесс с плотностью $\widetilde{\rho}(z, y)$.

Пусть $p(z, s)$ является условной плотностью $X_{s}$ при условии $\xi_{\tau}, \tau \in[s,+\infty)$. Тогда $p(z, s)$ п.н. удовлетворяет уравнению (16) (см. [17]), и процесс $\eta$, определенньй в (26), допускает представление

$$
\eta(s)=\mathrm{E}\left\{\operatorname{div}\left[a\left(X_{s}, \xi_{s}\right)\right]+b^{i}\left(X_{s}, \xi_{s}\right)-\bar{b}^{i} \mid \sigma\left(\xi_{\tau}, \tau \geqslant s\right)\right\} .
$$

Отметим, что условие (49) эквивалентно равенству $\mathrm{E} f\left(\widetilde{X}_{s}, \zeta_{s}\right)=0$. В частности, условие разрешимости вьполнено для функции $f^{b}(z, y)=\left(\operatorname{div}_{z}[a(z, y)]+b^{i}(z, y)-\bar{b}^{i}\right)$. Обозначим соответствуюшее решение символом $\chi_{b}(z, y)$. Применяя формулу Ито к выражению $\chi_{b}\left(\widetilde{X}_{s}, \zeta_{s}\right)$, после простых преобразований получим

$$
\begin{aligned}
\int_{0}^{t} f^{b}\left(\widetilde{X}_{s}, \zeta_{s}\right) d s= & \chi_{b}\left(\widetilde{X}_{t}, \zeta_{t}\right)-\chi_{b}\left(\widetilde{X}_{0}, \zeta_{0}\right) \\
& +\int_{0}^{t} \sigma\left(\widetilde{X}_{s}, \zeta_{s}\right) \nabla_{z} \chi_{b}\left(\widetilde{X}_{s}, \zeta_{s}\right) d w_{s}^{1}+\int_{0}^{t} \Sigma\left(\zeta_{s}\right) \nabla_{y} \chi_{b}\left(\widetilde{X}_{s}, \zeta_{s}\right) d w_{s}^{2}
\end{aligned}
$$

где $w^{1}$ и $w^{2}$ - независимые винеровские процессы размерности $n$ и $d$ соответственно и

$$
\sigma(z, y)=\sqrt{\left\{a_{i j}(z, y)\right\}}, \quad \Sigma(y)=\sqrt{\left\{q_{k l}(y)\right\}} .
$$

Взяв в предыдушей формуле условное математическое ожидание относительно $\sigma$-алгебры $\widetilde{\mathscr{F}}_{-\infty, t}=\sigma\left\{\zeta_{\tau},-\infty<\tau \leqslant t\right\}=\mathscr{F}_{-t,+\infty}$ и приняв во внимание независимость $\{\zeta$.$\} и w^{1}$, найдем:

$$
\begin{aligned}
\frac{1}{\sqrt{t}} \int_{0}^{t} \eta_{-s} d s= & \frac{1}{\sqrt{t}} \mathrm{E}\left\{\chi_{b}\left(\widetilde{X}_{t}, \zeta_{t}\right) \mid \widetilde{\mathscr{F}}_{-\infty, t}\right\} \\
& +\frac{1}{\sqrt{t}} \mathrm{E}\left\{\int_{0}^{t} \Sigma\left(\zeta_{s}\right) \nabla_{y} \chi_{b}\left(\widetilde{X}_{s}, \zeta_{s}\right) d w_{s}^{2} \mid \widetilde{\mathscr{F}}_{-\infty, t}\right\} \\
= & \frac{1}{\sqrt{t}} \mathrm{E}\left\{\chi_{b}\left(\widetilde{X}_{t}, \zeta_{t}\right) \mid \widetilde{\mathscr{F}}_{-\infty, t}\right\} \\
& +\frac{1}{\sqrt{t}} \int_{0}^{t} \mathrm{E}\left\{\Sigma\left(\zeta_{s}\right) \nabla_{y} \chi_{b}\left(\widetilde{X}_{s}, \zeta_{s}\right) \mid \widetilde{\mathscr{F}}_{-\infty, s}\right\} d w_{s}^{2}
\end{aligned}
$$

где $\eta_{s}$ это процесс, заданньй в $(26)$.

Первьй член в правой части стремится к нулю, когда $t \rightarrow \infty$. Вычисляя квадратичную характеристику интеграла Ито во втором члене и используя теорему Биркгофа, имеем

$$
\begin{aligned}
& \frac{1}{t} \int_{0}^{t} q\left(\zeta_{s}\right) \mathrm{E}\left\{\nabla_{y} \chi_{b}^{i}\left(\widetilde{X}_{s}, \zeta_{s}\right) \mid \widetilde{\mathscr{F}}_{-\infty, s}\right\} \mathrm{E}\left\{\nabla_{y} \chi_{b}^{j}\left(\widetilde{X}_{s}, \zeta_{s}\right) \mid \widetilde{\mathscr{F}}_{-\infty, s}\right\} d s \\
& \rightarrow \mathrm{E}\left(q\left(\zeta_{0}\right) \mathrm{E}\left\{\nabla_{y} \chi_{b}^{i}\left(\widetilde{X}_{0}, \zeta_{0}\right) \mid \widetilde{\mathscr{F}}_{-\infty, 0}\right\} \mathrm{E}\left\{\nabla_{y} \chi_{b}^{j}\left(\widetilde{X}_{0}, \zeta_{0}\right) \mid \widetilde{\mathscr{F}}_{-\infty, 0}\right\}\right) \\
& \quad=\mathrm{E}\left(q\left(\xi_{0}\right) \mathrm{E}\left\{\nabla_{y} \chi_{b}^{i}\left(\widetilde{X}_{0}, \xi_{0}\right) \mid \mathscr{F}_{0,+\infty}\right\} \mathrm{E}\left\{\nabla_{y} \chi_{b}^{j}\left(\widetilde{X}_{0}, \xi_{0}\right) \mid \mathscr{F}_{0,+\infty}\right\}\right)=\left(\Lambda^{2}\right)^{i j}
\end{aligned}
$$


Наконец, согласно [18; теоремы 9.1, 9.2] имеет место сходимость

$$
\frac{1}{\sqrt{\tau}} \int_{0}^{t \tau} \eta_{-s} d s \underset{\tau \rightarrow \infty}{\stackrel{\mathscr{L}}{\longrightarrow}} \Lambda W_{t}
$$

где $W_{t}-$ стандартньй $n$-мерный винеровский процесс.

\section{СПИСОК ЛИТЕРАТУРЫ}

[1] D. G. Aronson. Bounds for the fundamental solutions of a parabolic equation // Bull. Amer. Math. Soc. 1967. V. 73. P. 890-896.

[2] M. Avellaneda, A. J. Majda. Mathematical models with exact renormalization for turbulent transport // Comm. Math. Phys. 1990. V. 131. № 2. P. 381-429.

[3] M. Avellaneda, A. J. Majda. Simple examples with features of renormalization for turbulent transport // Philos. Trans. Roy. Soc. London Ser. A. 1994. V. 346. № 1679. P. 205-233.

[4] A. Bensoussan, J.-L. Lions, G. Papanicolaou. Asymptotic Analysis for Periodic Structures. Amsterdam: North-Holland, 1978. (Stud. Math. Appl. V. 5.)

[5] П. Биллингсли. Сходимость вероятностных мер. М.: Наука, 1977.

[6] R. Bouc, E. Pardoux. Asymptotic analysis of p.d.e.s with wide-band noise disturbances, and expansion of the moments // Stochastic Anal. Appl. 1984. V. 2. № 4. P. 369-422.

[7] F. Campillo, M. Kleptsyna, A. Piatnitski. Homogenization of random parabolic operator with large potential // Stochastic Process. Appl. 2001. V. 93. № 1. P. 57-85.

[8] G. Da Prato, J. Zabczyk. Stochastic Equations in Infinite Dimensions. Cambridge: Cambridge Univ. Press, 1992. (Encyclopedia Math. Appl. V. 44.)

[9] A. Fannjiang, G. Papanicolaou. Convection enhanced diffusion for periodic flows // SIAM J. Appl. Math. 1994. V. 54. № 2. P. 333-408.

[10] A. Fannjiang, G. Papanicolaou. Diffusion in turbulence // Probab. Theory Related Fields. 1996. V. 105. № 3. P. 279-334.

[11] J. Garnier. Homogenization in a periodic and time-dependent potential // SIAM J. Appl. Math. 1997. V. 57. № 1. P. 95-111.

[12] V. Jikov, S. Kozlov, O. Oleinik. Homogenization of Differential Operators and Integral Functionals. Berlin: Springer-Verlag, 1994.

[13] M. Kleptsyna, A. Piatnitski. Homogenization of random parabolic operators // Homogenization and Applications to Material Sciences: Proceedings of the International Conference, Nice, 1995. Tokyo: Gakkōtosho, 1997. P. 241-255. (GAKUTO Internat. Ser. Math. Sci. Appl. V. 9.)

[14] С. М. Козлов. Приводимость квазипериодических дифференциальных операторов и усреднение // Труды ММО. 1983. Т. 46. С. 99-123.

[15] С. М. Козлов, А. Л. Пятницкий. Усреднение на фоне исчезающей вязкости // Матем. сб. 1990. Т. 181. №6. С. 813-832.

[16] О. А. Ладыженская, В.А. Солонников, Н. Н. Уральцева. Линейные и квазилинейные уравнения параболического типа. М.: Наука, 1967.

[17] Р. Ш. Липцер, А. Н. Ширяев. Статистика случайных процессов. М.: Наука, 1974.

[18] Р. Ш. Липцер, А. Н. Ширяев. Теория мартингалов. М.: Наука, 1986.

[19] E. Pardoux. Homogenization of linear and semilinear second order parabolic PDEs with periodic coefficients: a probabilistic approach // J. Funct. Anal. 1999. V. 167. № 2. P. 498-520.

[20] E. Pardoux, A. Yu. Veretennikov. On the Poisson equation and diffusion approximation. I // Ann. Probab. 2001. V. 29. № 3. P. 1061-1085.

[21] А. Л. Пятницкий. Усреднение сингулярно возмущенного уравнения с быстроосциллирующими коэффициентами в слое // Матем. сб. 1983. Т. 121. № 1. С. 18-39.

[22] N.S. Trudinger. Pointwise estimates and quasilinear parabolic equations // Comm. Pure Appl. Math. 1968. V. 21. P. 205-226. 
[23] M. Viot. Solutions Faibles d'Équations Dérivées Stochastiques Non Linéaires // Thèse. Université Paris VI, 1976.

[24] В.В.Жиков. Замечания к проблеме остаточной дифффузии // УМН. 1989. Т. 44. №6. C. $155-156$.

[25] В. В. ЖКиков. Диффузия в несжимаемом случайном потоке // Функц. анализ и его прил. 1997. T. 31. № 3. С. $10-22$

Институт проблем передачи информации РАН;

Поступила в редакцию Технологический институт Нарвика, Норвегия, 05.04 .2002 Физический институт им. П. Н. Лебедева РАН

E-mail: Masha.Kleptsyna@id.ru, andrey@sci.lebedev.ru 